\title{
A Comparative Study of Helicopter Planetary Bearing Diagnosis with Vibration and Acoustic Emission Data
}

\author{
Linghao Zhou, Fang Duan, David Mba \\ School of Engineering \\ London South Bank University \\ London, U. K. \\ Zhoul7@1sbu.ac.uk
}

\author{
Elasha Faris \\ School of Mechanical, Automotive and Aerospace \\ Coventry University \\ Coventry, U.K. \\ ac1027@coventry.ac.uk
}

\begin{abstract}
Planetary bearings inside a helicopter main gearbox (MGB) are key components, successful diagnosis of planetary bearing defects benefits helicopter maintenance, reducing accidents rate and increasing aircraft flightworthiness. Most widely adopted methods for bearing fault diagnosis are envelope analysis and kurtogram; both have achieved many successes in practical application. However, diagnosis of faulty planetary bearing inside a MGB can be more complicated for reasons such as extremely strong operating noise, overwhelming gear meshes and so on. In this paper, acoustic emission (AE) data was recorded in comparison with traditional vibration data from test rig built using commercial helicopter gearbox with seeded defects, diagnosis of vibration data and $\mathrm{AE}$ data is performed using kurtogram and envelope analysis, results showed that $\mathrm{AE}$ signals are more sensitive to excitations from defects and suffer less from background noises.
\end{abstract}

Keywords- helicopter main gearbox; planetary bearing diangosis; acoustic emission signal; kurtogram; envelope analysis

\section{INTRODUCTION}

Bearings are one of the most crucial components in a helicopter main gearbox (MGB). MGB reduces high input speed generated from engines, hence providing low output speed with high torque to drive the main rotors [1]. Usually the speed reduction rate is so large that strong stress and forces are applied to planetary bearings, making them prone to all kinds of defects including bearing pitting, spalling and contact wear [2]. Helicopter health and usage monitoring system (HUMS) was first developed during 1990s and installed on medium and large civil helicopters to monitor aircraft flight status [3]. HUMS collects helicopters vibration data during specific flight regimes, and processes the data at Signal Processing Unit (SPU), generating condition indicators (CIs). Most CIs have pre-set thresholds which trigger the system alarms once being breached. More detailed data analysis however, is performed at ground base station, with data stored at a storage unit [4].

Installation of HUMS has evidently reduced accidents rate, according to a survey carried out by US Joint Helicopter Safety Implementation Team [4]. But as a continuously evolving system, HUMS is still not completely accurate and reliable for incipient fault detection. Some tragic helicopter crashes happened in recent decade indicate that HUMS failed to diagnose incipient defects happened on planetary bearings inside MGB before flight. During flight small cracks propagated and developed across MGB case, thus eventually led to rotor imbalance and aircraft disassembly [5-6].

Multiple reasons could contribute to HUMS not being able to react properly to incipient planetary bearing defects, including but not limited to:

- Epicyclic Modules of MGB can achieve a large reduction rate of approximately 86:1 (calculated based on test rig MGB). Such functionality is realised by a sophisticated mechanical structure, which involves many planetary gears and bearings (see Fig. 1 and Fig. 2 ). This structure complicates signal transmission paths for accelerometers, hence vibration data collected are highly amplitude and frequency modulated, which potentially masks faulty signature.

- Decided by the structure of planetary gear/ bearing set, gears and bearings share same races, which result in overwhelming gear meshes signal masking planetary bearing signals that are inherently weak. Thus faulty signatures excited by contacts between defects and rollers/ cages are difficult to be extracted.

- An operating helicopter MGB, especially in high-speed mode, generates extremely large noise from the frictions and contacts between MGB components. Signal-to-noise (SNR) ratio is not idea enough for direct clear diagnosis under such harsh circumstances.

- Traditional HUMS CIs are generated based on statistical characteristics of vibration data, and sometime interpreting statistical features can be tricky. For example, kurtosis can be used to describe shape of the probability distribution of the vibration data, i.e. how flat or steep the data are. However kurtosis will lose its validity once the initial defects propagate into more distributed or severed defects. In addition, low SNR affects CIs' accuracy drastically. 
Traditional vibration-based monitoring suffers from interferences from strong noise and the insensitivity to incipient defects. In recent years, acoustic emission (AE) based monitoring has drawn researchers' attention, since $\mathrm{AE}$ has great potentials of being a supplementary monitoring technique [7]. AE is defined as an elastic wave, generated when changes occur inside or on the surface of the monitored materials. AE signals have high frequency contents. Commonly an effective AE signal frequency bandwidth could range from $100 \mathrm{kHz}$ to several megahertz. Audible operational noise (bandwidth below $20-22 \mathrm{kHz}$ ) will be insignificant in AE signal bandwidth. $\mathrm{AE}$ is also very sensitive to changes inside or on the surface of monitored materials, including collisions induced by defects inside planetary bearings [8]. AE has been reported numerously to be successfully applied for structural health monitoring [910], e.g. bridges and walls cracks detection. But for monitoring a rotational machine, there are still many concerns. First of all, $\mathrm{AE}$ sensor ideally needs be as close as possible to the $\mathrm{AE}$ sources, because AE attenuates rapidly. Thus AE sensor should be attached close to rotating bearings, which requires the $\mathrm{AE}$ transmission system to be wireless. Secondly, high frequency contents can only be properly recorded with a sampling rate of at least twice the largest frequency components, according to Nyquist Sampling Theorem. This means at least $2 \mathrm{MHz}$ or even higher sampling frequency shall be adopted, which makes transmitting digitalised AE data difficult to accomplish.

To address these issues, a wireless AE transmission system is designed in [11], using homodyne "back scattering" structure, that transferring analogue AE signal from rotating components directly, and then digitalised at stationary PC. The design of $\mathrm{AE}$ transmission system is described in detail in [11] and [12].

To validate the application of AE signal on MGB planetary bearing fault diagnosis, and compare the effectiveness of diagnosis using $\mathrm{AE}$ and vibration data, an experiment was undertaken using a commercial "Category A" helicopter MGB, model type SA 330 puma. More details will be described in next section.

\section{EXPERIMENT SETUP}

\section{A. $\quad$ Test Procedures}

The MGB adopted in this test comprised of two epicyclic modules, a forward-module connected to a high speed DC motor, and an after-module that was left idle. 9 planetary bearings were installed at $2^{\text {nd }}$ epicyclic module, and defects of two different sizes were planted at the outer race of planetary bearings $\left(2^{\text {nd }}\right.$ planetary bearings are shown in Fig. 1), namely "minor defect" and "major defect", with dimensions of $10 \mathrm{~mm}$ wide, $0.3 \mathrm{~mm}$ deep and $30 \mathrm{~mm}$ wide, $0.3 \mathrm{~mm}$ deep respectively. The major defect was created that the defect range covered approximately $40^{\circ}$ of the entire outer ring. An illustration of major defect seeded at planetary bearing is shown in Fig. 3. An adjustable load is added on top of MGB $2^{\text {nd }}$ planetary carrier to simulate the main rotor.

To fully test the MGB and push the operational limits, the extreme test condition was simulated by inputting the rig with

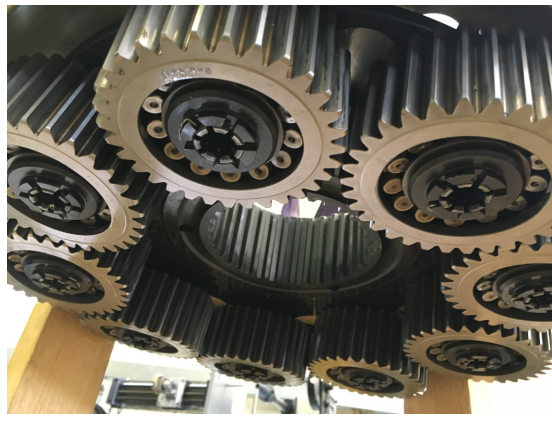

Figure 1. Demonstration of $2^{\text {nd }}$ planetary gear/ bearing set

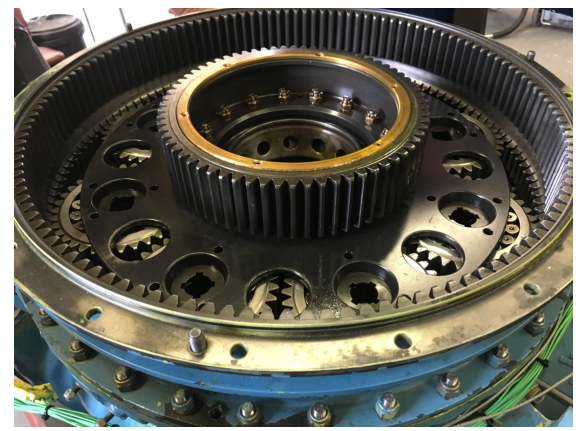

Figure 2. Demonstration of $2^{\text {nd }}$ planetary sun gear and ring gear

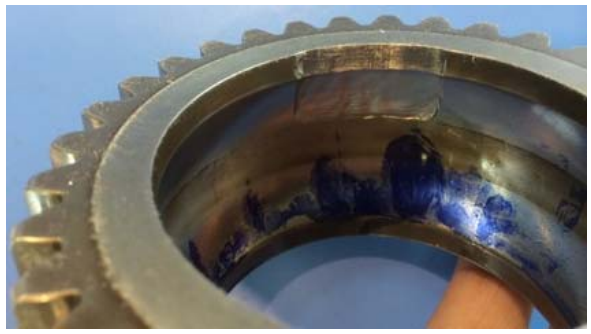

Figure 3. Major defect seeded at planetary bearing outer race

24,000rpm speed, getting 275rpm output speed, and continuously ran the rig for 20 minutes under the major fault condition with $1760 \mathrm{~kW}$ load. This load is $110 \%$ of the power that the helicopter requires for take-off. Other test conditions include power ranging from $1600 \mathrm{~kW}$ to $936 \mathrm{~kW}$, under both major and minor fault condition. Detailed experiments arrangement is described in [12].

\section{B. Data Recording}

Vibration data were recorded using accelerometers with sensitivity of $10 \mathrm{mV} / \mathrm{g}$, positioned at 6 different locations. Higher sensitivity of $100 \mathrm{mV} / \mathrm{g}$ was not applicable as the level of vibration generated from an operating MGB could easily saturate the sensor. In order to capture vibration details as much as possible, data sampling frequency of $51.2 \mathrm{kHz}$ was selected, and a $25.6 \mathrm{kHz}$ anti-aliasing filtering was applied before storing data.

For AE data capturing, the afore-described wireless system was installed at $2^{\text {nd }}$ epicyclic module carrier plate, which was above the faulty bearing. A miniature PWAS AE sensor was 
adopted. After receiving AE from outside of the epicyclic case, the signal was sampled at $5 \mathrm{MHz}$, no filtering was applied.

\section{SIGNAL PROCESSING}

\section{A. Fault Frequency Extraction Based Diagnosis}

It is well recognised that data in the form of time series most commonly reveal little diagnosis information. This is especially true for planetary bearing signals, because of complicated amplitude modulation and transmission paths. Fast Fourier Transform (FFT) is designed to convert signals in time domain to frequency domain, where periodic signatures of the signal are exposed, thus FFT has always been adopted extensively when dealing with signals containing periodic information. Assume a scenario where MGB maintains a constant rotating speed. As planetary bearing rolling, faulty bearing rollers continuously collide on defects at bearing outer race, producing periodic spikes which can be reflected in frequency spectrum as a component called "ball pass frequency of outer race" (BPFO). BPFO theoretically is very tiny or not observable under healthy condition. Thus a distinctive comparison can be made between faulty condition and healthy condition. Equations of calculating BPFO and many other relevant faulty frequencies can be found in many sources [13].

The essential idea adopted in this paper is based on traditional fault frequency extraction method, i.e. identifying the existence of defects by examining the signal in frequency domain for matches of stand-out fault frequencies. Major fault and minor fault diagnosis results under conditions of high input speed, high load are presented in this paper. The summarised key parameters are:

TABLE I. KEY PARAMETERS

\begin{tabular}{|c|c|c|c|}
\hline \multirow{2}{*}{$\begin{array}{c}\text { All } \\
\text { conditions }\end{array}$} & \multicolumn{3}{|c|}{ Key Parameters } \\
\cline { 2 - 4 } & Input Speed & Output Speed & BPFO \\
\hline Input Speed & $23688 \mathrm{RPM}$ & $274.8 \mathrm{RPM}$ & $100.3 \mathrm{~Hz}$ \\
\hline
\end{tabular}

\section{B. Envelope Analysis}

In practical, application of direct FFT on bearing fault diagnosis cannot be as effective as expected, due to reasons discussed in Section. 1. To date, most widely adopted benchmark method for bearing fault diagnosis is envelope analysis [14]. As the bearing roller colliding defects, the structure of bearing amplifies such impact and produces high frequency resonances which are amplitude modulated by the faulty signals while gear meshes are less dominating. SNR of bearing signals within structural resonances' bandwidth could be high enough for diagnosis tasks. Envelope analysis thus targets high frequency structure resonances, using band-pass filter to capture this particular part and then demodulate it by acquiring its modulated amplitude envelope. FFT of the envelope is at last performed to reveal the repetitive nature of bearing faulty signals. In practical, gear meshes still exist in vibration envelope spectrum, thus some pre-processing of gear/ bearing signal separation techniques are suggested to be applied before envelope analysis. J. Antoni and B. Randall have published numerous researches on such topics [14-17].

\section{Kurtogram}

Put aside all the advantages of envelope analysis, one drawback is the difficulty of determining centre frequency of structural resonances and band-pass bandwidth. Traditionally, researchers use a hammer tapping on the rig before actual tests to acquire structure resonances [18], which is not efficient.

J. Antoni adopted the concept of spectral kurtosis (SK), which is defined as kurtosis of complex random variable at each frequency bin [19], and developed kurtogram to properly demonstrate SK by calculating SK in different frequency bandwidths and centre frequency [20]. Applying kurtogram can effectively extract information of most impulsive centre frequency and its frequency band which possibly contains bearing faulty signature. The discovered centre frequency and bandwidth are then utilised in envelope analysis.

Since AE signals are also continuously amplitude modulated signal, kurtogram and envelope analysis are applicable as well. Results and comparisons are discussed in next section.

\section{RESULTS AND DISCUSSION}

\section{A. Vibration Data Processing}

Kurtogram was first applied on the vibration data, after some denoising pre-processing.

The kurtogram result suggested resonances at approximately $22000 \mathrm{~Hz}$ centre frequency with $8000 \mathrm{~Hz}$ bandwidth. By contrast, the same area in the amplitude spectrum showed structural resonances pattern. Envelope analysis using selected frequency parameters was performed

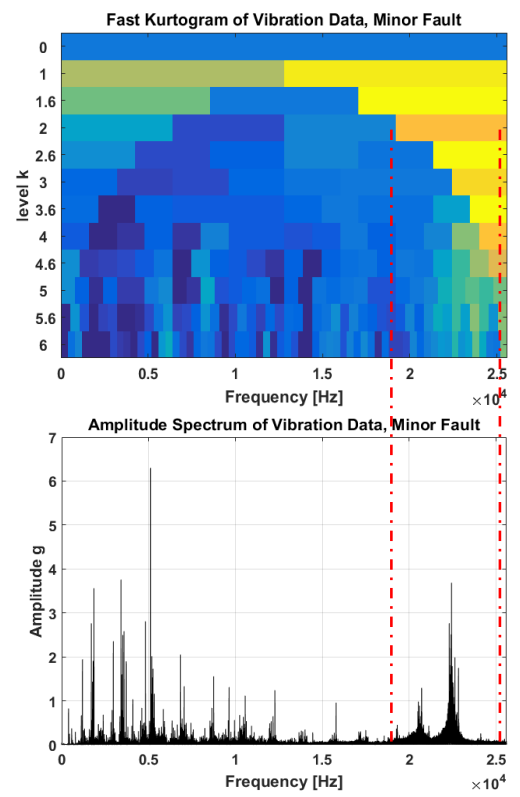

Figure 4. Kurtogram of vibration data, incomparison of FFT, minor fault 
(for all three conditions), results shown in Fig. 5.
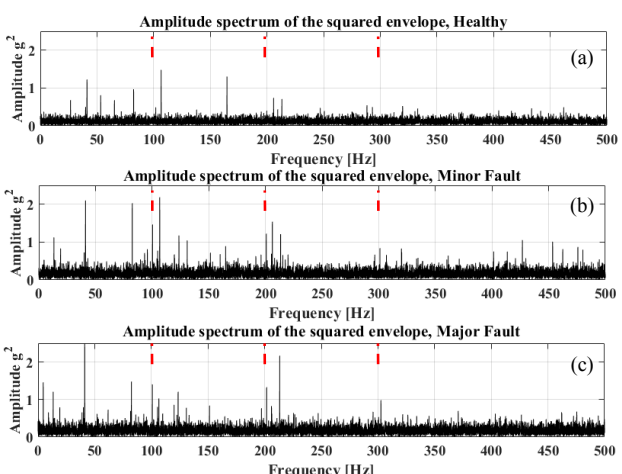
Minor Fault and (c) Major Fault
Figure 5. Vibration Envelope analysis results, (a) Healthy (b)

analysis results were dominating. No suspicious frequency components were large enough to affect the identification of defects; moreover, the defect severity progress was also reflected as BPFO of major defect was significantly larger than that of minor condition.

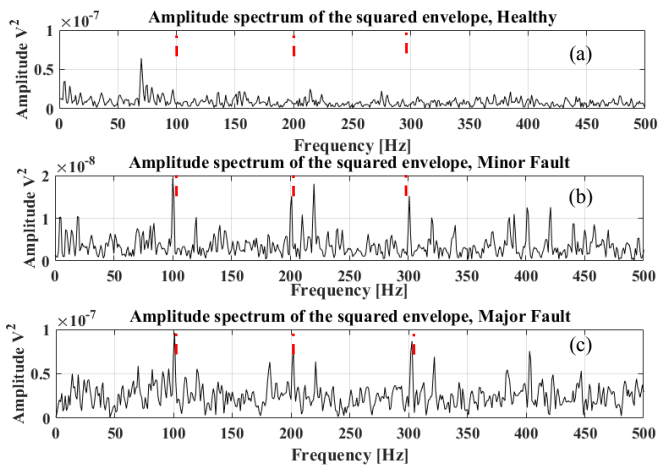

Figure 7. AE Envelope analysis results, (a) Healthy (b) Minor Fault and (c) Major Fault

\section{CONCLUSIONS}

It is evident that under healthy condition, very tiny frequency components near $100 \mathrm{~Hz}$ can be observed, no corresponding $2^{\text {nd }}$ and $3^{\text {rd }}$ harmonics of such frequency can be observed. In both minor fault and major fault condition, distinctive $100 \mathrm{~Hz}$ BPFO components can be identified, as well as the $2^{\text {nd }}$ and $3^{\text {rd }}$ harmonics, indicating a fault related signal signature.

Nonetheless, disturbing frequency components and harmonics were still obvious in Fig. 5. For example the strong $106 \mathrm{~Hz}$ frequency components existed in all three conditions. This suspicious frequency and its sidebands, harmonics can hinder the accuracy of fault detection under low SNR conditions.

\section{B. AE Data Proessing}

Similar processing routine was applied at AE data. The results are shown below:

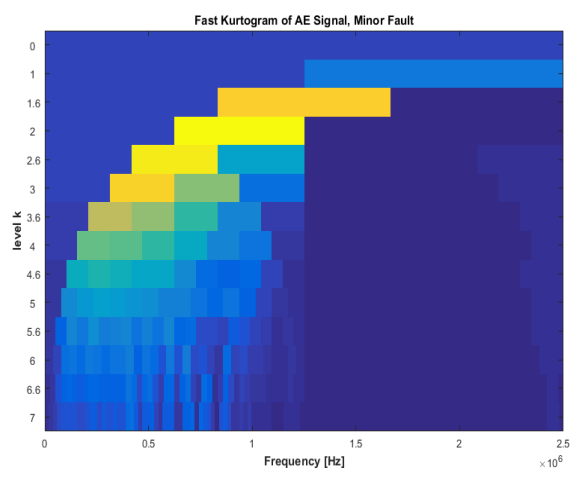

Figure 6. Direct kurtogram of AE signal

Result in Fig. 7 suggested a clear indication of faulty BPFO frequency components near $100 \mathrm{~Hz}$ in both minor and major condition. BPFO and its $2^{\text {nd }}$ and $3^{\text {rd }}$ harmonics in AE envelope
The diagnosis regarding commercial MGB with seeded defects was successful using kurtogram and envelope analysis. However, AE data have shown some great features compared with traditional vibration data. According to Fig. 5 and Fig. 7, AE data were less sensitive to the modulation from $2^{\text {nd }}$ epicyclic carrier speed and gear meshes, which showed the superiority of the AE system installed. Also AE results revealed the severity of defects, which was not reflected in vibration data processing results. The high frequency content of AE naturally blocked the interferences from ambient noises, which potentially increased SNR and benefits fault diagnosis. In summary, $\mathrm{AE}$ signal monitoring technique has potentials to improve HUMS detection capability for bearing defects.

\section{REFERENCES}

[1] D. Mba, S. Place, H. Rashid, and C. Lim, "Helicopter main gearbox loss of oil performance optimization-HELMGOP," vol. 5, 2011.

[2] L. Frosini and E. Bassi, "Stator current and motor efficiency as indicators for different types of bearing faults in induction motors," vol. 57, no. 1, pp. 244-251, 2010.

[3] J. E. Land, "HUMS-the benefits-past, present and future," IEEE, 2001, vol. 6, pp. 3083-3094.

[4] L. Kaufman and J. Gregoire, "Health and usage monitoring systems Toolkit courtesy of American Eurocopter," 2013. [Online]. Available: http://www.ihst.org/portals/54/toolkit_hums.pdf. Accessed: Feb. 17, 2017

[5] M. Jarvis and P. Sleight, "Report on the accident to aerospatiale (eurocopter) AS332 L2 super puma registration G-REDL," vol. 2, no. 2011, p. 24, 2011.

[6] A.-L. Bjella-Fosshaug, "Investigation of helicopter accident at Turøy near Bergen in Hordaland county, Norway," 2015. [Online]. Available: https://www.aibn.no/Aviation/Investigations/16-286. Accessed: Feb. 17, 2017.

[7] V. Kostopoulos, T. Loutas, and K. Dassios, "Fracture behavior and damage mechanisms identification of $\mathrm{SiC} /$ glass ceramic composites using AE monitoring," vol. 67, no. 7, pp. 1740-1746, 2007. 
[8] A. M. Al-Ghamd and D. Mba, "A comparative experimental study on the use of acoustic emission and vibration analysis for bearing defect identification and estimation of defect size," vol. 20, no. 7, pp. 1537$1571,2006$.

[9] A. Nair and C. Cai, "Acoustic emission monitoring of bridges: Review and case studies," vol. 32, no. 6, pp. 1704-1714, 2010.

[10] A. Farhidzadeh, S. Salamone, B. Luna, and A. Whittaker, "Acoustic emission monitoring of a reinforced concrete shear wall by b-valuebased outlier analysis," vol. 12, no. 1, pp. 3-13, 2013.

[11] M. Greaves, "Towards the next generation of HUMS sensor," in ISASI 2014 Seminar, Adelaide, Australia, 2014.

[12] F. Elasha, M. Greaves, D. Mba, and A. Addali, "Application of Acoustic Emission in Diagnostic of Bearing Faults within a Helicopter Gearbox," vol. 38, pp. 30-36, 2015.

[13] L. Eren and M. J. Devaney, "Bearing damage detection via wavelet packet decomposition of the stator current," vol. 53, no. 2, pp. 431-436, 2004.

[14] [R. B. Randall and J. Antoni, "Rolling element bearing diagnostics - a tutorial," vol. 25, no. 2, pp. 485-520, 2011.
[15] J. Antoni and R. Randall, "Unsupervised noise cancellation for vibration signals: part I-evaluation of adaptive algorithms," vol. 18, no. 1, pp. 89-101, 2004.

[16] J. Antoni and R. Randall, "Unsupervised noise cancellation for vibration signals: part II - a novel frequency-domain algorithm," vol. 18, no. 1, pp 103-117, 2004.

[17] R. B. Randall, "A history of cepstrum analysis and its application to mechanical problems," 2016.

[18] P. McFadden and J. Smith, "Vibration monitoring of rolling element bearings by the high-frequency resonance technique-a review," vol. 17, no. 1, pp. 3-10, 1984.

[19] V. Vrabie, P. Granjon, and C. Serviere, "Spectral kurtosis: from definition to application," 2003, p. xx.

[20] J. Antoni and R. Randall, "The spectral kurtosis: application to the vibratory surveillance and diagnostics of rotating machines," vol. 20, no. 2, pp. 308-331, 2006 